\title{
DESIGNING A ROUTING PROTOCOL FOR UBIQUITOUS NETWORKS USING ECA SCHEME
}

\author{
Chandrashekhar Pomu Chavan and Pallapa Venkataram \\ Protocol Engineering and Technology Unit, Department of Electrical \\ Communication Engineering, Indian Institute of Science, Bangalore, India \\ \{cpchavan, pallapa\} dece.iisc.ernet.in
}

\begin{abstract}
We have designed a novel Event-Condition-Action (ECA) scheme based Ad hoc On-demand Distance Vector(ECA-AODV) routing protocol for a Ubiquitous Network (UbiNet). ECA-AODV is designed to make routing decision dynamically and quicker response to dynamic network conditions as and when event occur. ECA scheme essentially consists of three modules to make runtime routing decision quicker. First, event module receive event that occur in a UbiNet and split up event into event type and event attributes. Second, condition module obtain event details from event module split up each condition into condition attributes that matches event and fire the rule as soon as condition hold. Third, action module make runtime decisions based on event obtained and condition applied. We have simulated and tested the designed ECA scheme by considering ubiquitous museum environment as a case study with nodes range from 10 to 100. The simulation results show the time efficient with minimal operations.
\end{abstract}

\section{KEYWORDS}

Ubiquitous Network, Routing, Event, Subnet, Topology

\section{INTRODUCTION}

Ubiquitous Network (UbiNet) is a heterogeneous network [1] with various computing devices which are connected at anywhere, anytime and enable users to access and exchange information [2]. In UbiNet, the nodes may join/leave the network frequently and move freely hence, leads to frequent change in network topology. Since the nodes are mobile they can move arbitrarily in any direction leads to various failures like link failure, node failure and so on. Hence the dynamic network topology and frequent failures can be addressed using a routing protocol to manage the variety of failures and to choose optimal paths to transmit the data [3].

Routing in UbiNet is to find a best path from user to the service provider. Basically, routing algorithms are designed to determine the best paths in the network, whereas routing table entries store route information that the algorithm has already discovered a best path and routing protocols allow data packet to be collected and distributed across the network [4]. A UbiNet do

David C. Wyld et al. (Eds) : ACITY, DPPR, VLSI, WiMNET, AIAA, CNDC - 2015

pp. 01-18, 2015. @ CS \& IT-CSCP 2015

DOI : $10.5121 /$ csit.2015.51301 
not support any existing routing protocols since a client node does not have(or any node) or do not maintain the routing tables[5], [6].

We develop an ECA scheme based routing protocol in UbiNet i.e. ECA-AODV routing protocol for making dynamic ubiquitous routing decision quickly at runtime. In an ECA scheme, event [7] is defined as significant changes in state of a system. Event can occur at any time, event module collect the detail information about the event and forward to condition module for applying logical procedure in order to make dynamic routing decision quickly. When a specific event is occurred at a particular time, certain conditions are met then action module make dynamic decision [8],[9],[10].

\subsection{Proposed Idea}

We have proposed an ECA scheme for routing protocol in UbiNet. An ECA scheme is broadly divided into three modules namely Event module, Condition module and Action module respectively. Event module is a 2-tuples consists of event types and event attributes, the function of event module is to keep observe and notifies events. Condition module is 2-tuples consists of event details and condition attributes.

Condition module observes and receives an event, look for the rule that matches inputs and fire the rule as soon as condition hold. Based on event and condition, a decision is made by the action module which contains 1-tuple action attributes. ECA scheme is store using structure data structure and distributed across every node. Upon receiving event at a particular node, node intern broadcast occurred event to all its vicinity nodes.

\subsection{Pattern of the Research Paper}

Pattern of the research paper is as follows. Section 2 present most relevant works. Section 3 briefly explains AODV routing. Section 4 briefly describes routing in UbiNet. Section 5 explain proposed ECA scheme. Section 6 describes the ubiquitous museum environment case study. Section 7 gives the simulation environment. 8 shows the simulation result and finally, the paper draws some conclusions in Section 9.

\section{SOME OF THE EXISTING WORKS}

Bhandari S.R. and Bergmann N.W [11] describes program do not respond well for component or resource failure. An ECA based system is suitable for both describing desired system operations as well as linking an event [12] based system to communicate with resources.

Hannes Obweger et al.[13] propose an innovative framework for creating sense and response rules that can be useful for real-time. Sense and response has set of rules to detect event [14] from business stream and take appropriate decision based on event occurrence.

Kaan Bur and Cem Ersoy [15] presents a novel mesh based QoS multicast routing which keep track of the resources availability at every node and monitor the QoS status periodically. QoS multicast routing will be selected on the basis of available resources, protocol selects optimal route in heterogeneous network. Gateway node does protocol conversion at the boundaries of the subnet. 
Jungil Heo and Wooshik Kim [16] analyze the information about user movement among heterogeneous subnet technologies in a ubiquitous system and proposes how to configure and manage the network in order to execute ubiquitous service in time. AODV protocol plays a vital role in health care environment to provide reliable and power efficiency data transmission.

Elizabeth M. Royer and Charles E. Perkins [17] consider fundamental functioning of AODV routing protocol in which, nodes store the route as and when needed. Each node uses destination sequence numbers to ensure freshness of the route at all times, AODV response quickly during link breakage in active routes.

\section{AODV ROUTING}

The AODV routing protocol is on-demand and reactive, in which path is discovered on-demand; established path is maintained as long as it is needed. AODV routing protocol [18] consists of four messages viz. i) Route request message, ii) Route reply message, iii) Route error message and iv) Route reply-acknowledgement message respectively. Essentially, node relies on intermediate nodes to find an optimal path from originating node to the ultimate target node in the network.

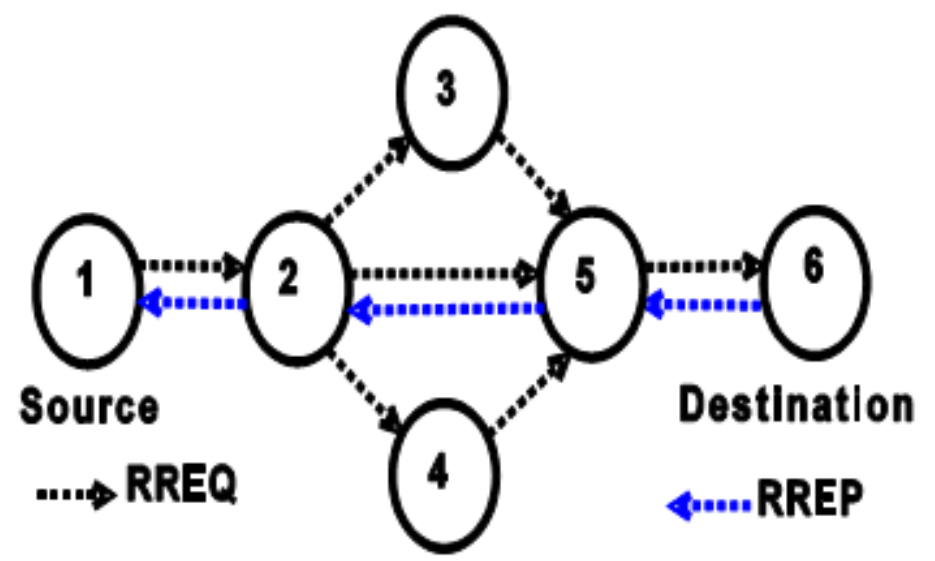

Figure 1. Route Discovery process in AODV Routing

In Figure 1, Node 1 has some data, it wishes to send to node 6 but node 1 does not have a valid path to communicate with node 6 , in such case node 1 initiate path finding by sending route request message to its vicinity node 2 , node 2 intern propagate the route request to its neighbour nodes such as 3, 4 and 5 respectively until route request reaches destination, if anyone of the intermediate node has the valid path toward the destination or node itself is destination may reply to the corresponding route request. Immediately upon receiving route request node verify that the sequence number [19] of the replying node is greater than that contained in route request message, then only node initiate to generate the route reply and unicast back to the route request originator. This is how a valid route is established in AODV routing. 


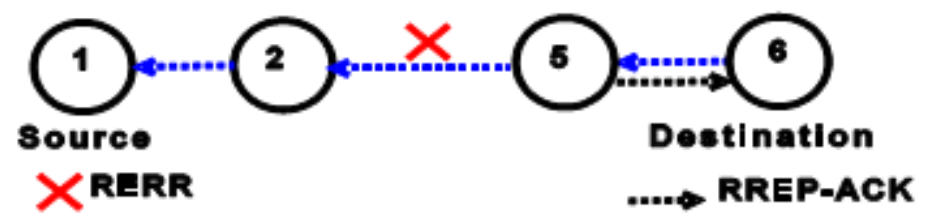

Figure 2. Route error and Route reply-ACK message

AODV uses route request and route reply for establishing a valid path, upon establishing valid path node store a routing table to communicate with rest of the nodes in the network, if existing path is not valid then node generate and forward the route error message to its predecessor node, when a node activate a bidirectional link then it send route reply acknowledgement message.
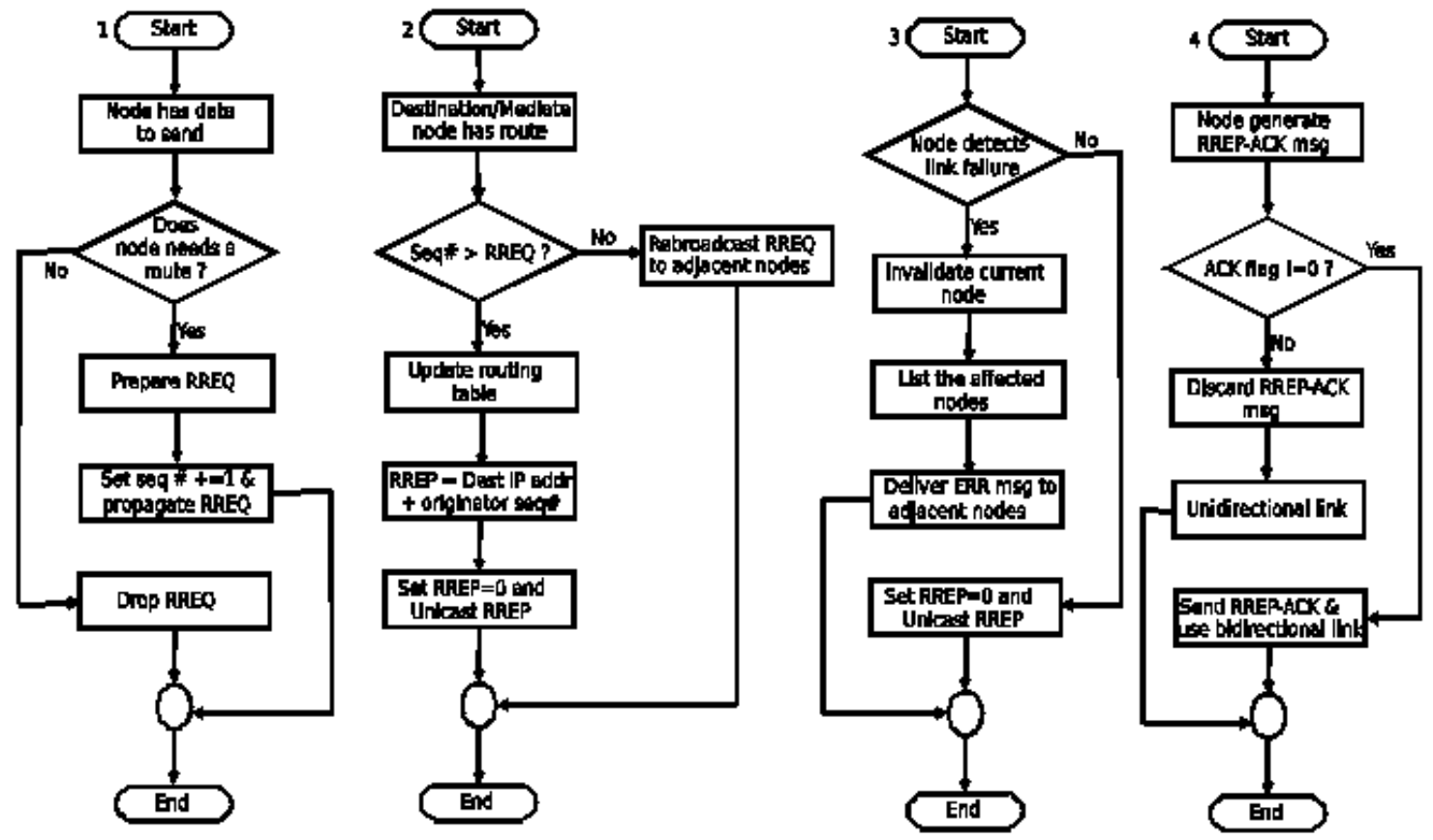

Figure 3. Flow chart of generating four AODV messages

Usually, in AODV originating node initiate a path discovery process as and when it would like to communicate with other node, node itself verify it has valid route or not if valid route does not exist then source node prepare RREQ packet and increment sequence number by one and propagate to its adjacent nodes.

\section{ROUTING IN UBIQUITOUS NETWORKS}

In ubiquitous environment, users demand constant availability of service at anytime from anywhere. Ubiquitous Server (UbiServ) receive informations about the user such as location, time, types of device, interest, preference, etc. from various embedded sensors and user profile, process the information and play a vital role in providing routing information to the user. UbiServ are connected and distributed across the internet, when a user enter into ubiquitous environment, an automatic path is establishes from user to the UbiServ without user's intervention [20]. 


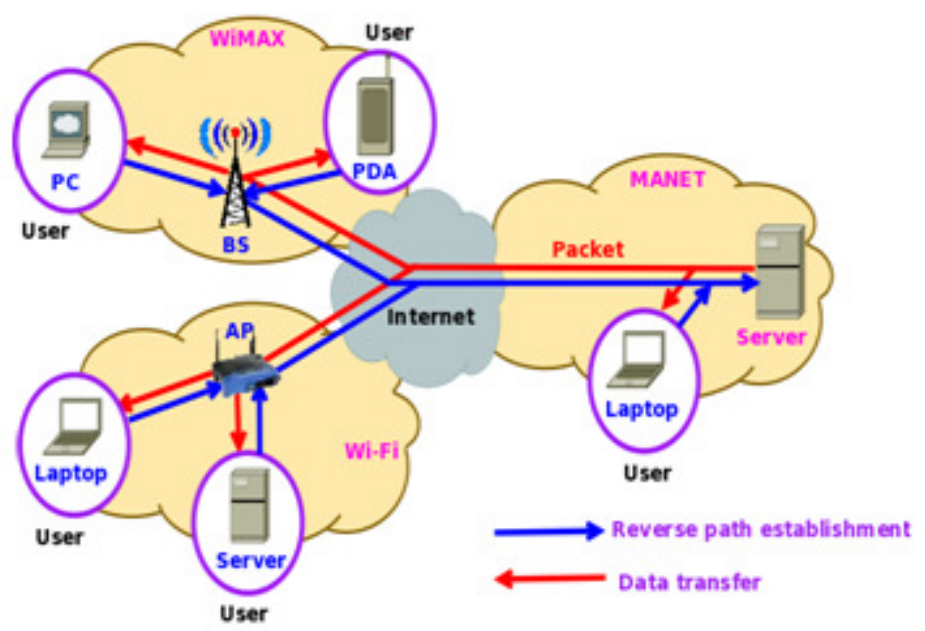

Figure 4. Example of routing in ubiquitous network

Routing in ubiquitous network has numerous differences as compared to normal routing in the following points

* Routing is adapted to heterogeneous network based on application requirements.

* UbiServ provide end-to-end flexible routing.

* Routing decision can be made dynamically based on current network status.

* Provide seamless service.

* Support QoS guaranteed service in high traffic.

* Lossless handover during switching from one network access technology to another network access technology.

* Provide best-effort traffic during congestion.

In ubiquitous network, a reverse path is established from user to the ubiquitous server, upon establishing path, UbiServ maintain an uninterrupted connectivity from user to the various subnet. UbiServ provide the routing information to the user located at heterogeneous subnet based on user's interest and preference.

\section{PROPOSED ECA SCHEME IN UbiNet}

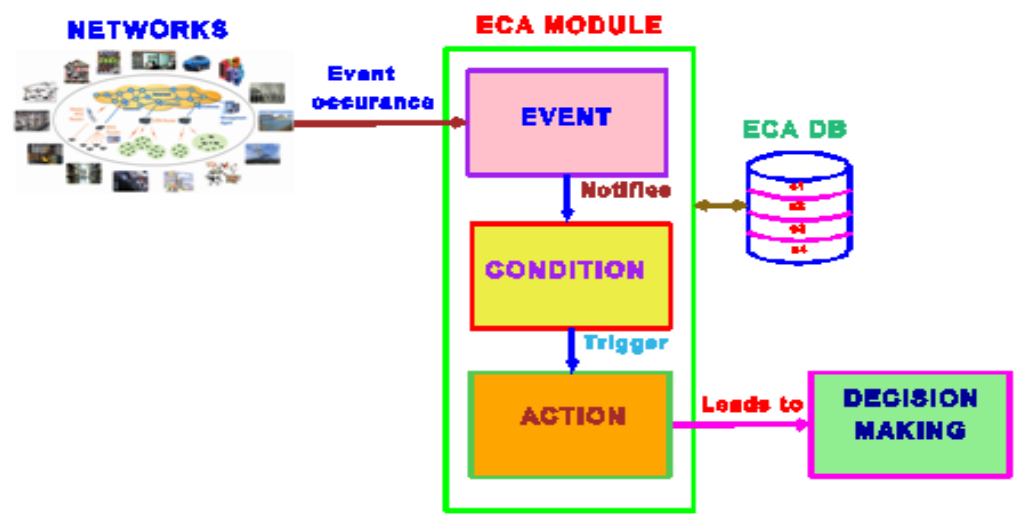

Figure 5. Function block diagram of an Event-Condition-Action Scheme 


\subsection{Function of Event Module}

Event is defined as significant changes in state of a system. Event module keep observe events and notifies as and when events occurs. Event is a 2-tuple consists of event types and event attributes, event is denoted by $E_{i}$ notation, event type may be time, spatial, composite, request, notification, internal, external, fault, service, etc.

$$
E_{i}=\left(t_{j}, a_{k}\right)
$$

Table 1. Event types

\begin{tabular}{|l|l|l|}
\hline \multicolumn{3}{|c|}{ Event types $\left(t_{\mathrm{j}}\right)$} \\
\hline Type 1 & Type $2 \ldots$ & Type $\mathrm{m}$ \\
\hline
\end{tabular}

Table 2. Event attribute

\begin{tabular}{|l|l|l|}
\hline \multicolumn{3}{|c|}{ Event attributes $\left(\mathrm{a}_{\mathrm{k}}\right)$} \\
\hline Data types & Parameters & Values \\
\hline
\end{tabular}

$E_{i}$ is the $i^{\text {th }}$ event, where $i \in\{1,2,3, \ldots, n\}, t_{j}$ is the $j^{\text {th }}$ event type, $a_{k}$ is the $k^{\text {th }}$ event attribute, $d a_{x}$ is the data type, $p_{y}$ is the parameter, $v_{z}$ is attribute value

$$
\begin{gathered}
\mathrm{t}_{\mathrm{j}} \in\left\{\mathrm{t}_{1}, \mathrm{t}_{2}, \mathrm{t}_{3}, \ldots, \mathrm{t}_{\mathrm{m}}\right\} \\
\mathrm{a}_{\mathrm{k}}=\left(\mathrm{da}_{\mathrm{x}}, \mathrm{p}_{\mathrm{y}}, \mathrm{v}_{\mathrm{z}}\right) \\
\mathrm{da}_{\mathrm{x}} \in\left\{\mathrm{da}_{1}, \mathrm{da}_{2}, \mathrm{da}_{3}, \ldots, \mathrm{da}_{\mathrm{k}}\right\} \\
\mathrm{p}_{\mathrm{y}} \in\left\{\mathrm{p}_{1}, \mathrm{p}_{2}, \mathrm{p}_{3}, \ldots, \mathrm{p}_{\mathrm{l}}\right\} \\
\mathrm{v}_{\mathrm{z}} \in\left\{\mathrm{v}_{1}, \mathrm{v}_{2}, \mathrm{v}_{3}, \ldots, \mathrm{v}_{\mathrm{x}}\right\}
\end{gathered}
$$

$E_{i}$ occurs at a particular time is given by $E_{i}(t)=\left\{\begin{array}{l}1 ; \text { Event has occured } \\ 0 ; \text { Otherwise }\end{array}\right.$

\subsection{Function of Condition Module}

Observe and receive an event, look for the rule that matches inputs and fire the rule as soon as condition hold. Condition module is 2-tuple consists of event details and condition attributes, $C_{l}=\left(d_{x}, c_{m}\right)$, Where $C_{l}$ is the $1^{\text {th }}$ condition, $d_{x}$ is the $x^{\text {th }}$ event details, $c_{m}$ is the $m^{\text {th }}$ condition attribute

$$
\mathrm{d}_{\mathrm{x}}=\left(\mathrm{E}_{\mathrm{i}}, \mathrm{t}_{\mathrm{j}}, \mathrm{a}_{\mathrm{k}}\right) \quad \text { and } \mathrm{c}_{\mathrm{m}}=\left(\mathrm{c}_{\mathrm{p}}, \mathrm{a}_{\mathrm{q}}, \mathrm{o}_{\mathrm{r}}, \mathrm{r}_{\mathrm{t}}\right)
$$

Table 3. Event details

\begin{tabular}{|c|}
\hline Event details $\left(\mathrm{d}_{\mathrm{x}}\right)$ \\
\hline $\mathrm{E}_{\mathrm{i}}, \mathrm{t}_{\mathrm{j}}, \mathrm{a}_{\mathrm{k}}$ \\
\hline
\end{tabular}


Table 4. Condition attributes

\begin{tabular}{|l|l|l|l|}
\hline \multicolumn{2}{|c|}{ Condition attributes $\left(\mathrm{c}_{\mathrm{m}}\right)$} \\
\hline Condition types & Arguments & Operators & Results \\
\hline
\end{tabular}

\subsection{Function of Action Module}

Based on event and condition an operation to be carried out.

Action is 1-tuple consists of action attributes.

Action $A_{n}=\left(a t_{p}\right)$, where $A_{n}$ is the $n^{\text {th }}$ action, at ${ }_{p}$ is the $p^{\text {th }}$ action attribute.

$>$ When $\left\{E_{i}\right\}$ occurs if $\left\{C_{1}\right.$ true $\}$ then $\left\{\right.$ Execute $\left.A_{n}\right\}$.

$>$ ECA scheme is distributed across the network i.e R: $\left\{\mathrm{E}_{\mathrm{i}}, \mathrm{C}_{1}\right\} \rightarrow \mathrm{A}_{\mathrm{n}}$.

Table 5. Action attributes

\begin{tabular}{|l|l|l|}
\hline \multicolumn{3}{|c|}{ Action attributes $\left(\mathrm{at}_{\mathrm{p}}\right)$} \\
\hline $\mathrm{E}_{\mathrm{i}}$ & Condition result & Decision making \\
\hline
\end{tabular}

Algorithm1: Algorithm for dynamic routing decision using ECA scheme

\section{1: Begin}

2: Input: Set of events

3: Output: Dynamic routing decision

4: if(Event has occurred in Ubiquitous network) then

5: $\quad$ Split every event into event type and event attributes

6: Apply logical condition

7: $\quad$ Make runtime decision

8: else

9: Do not make dynamic routing decision

10: end if

11: End 


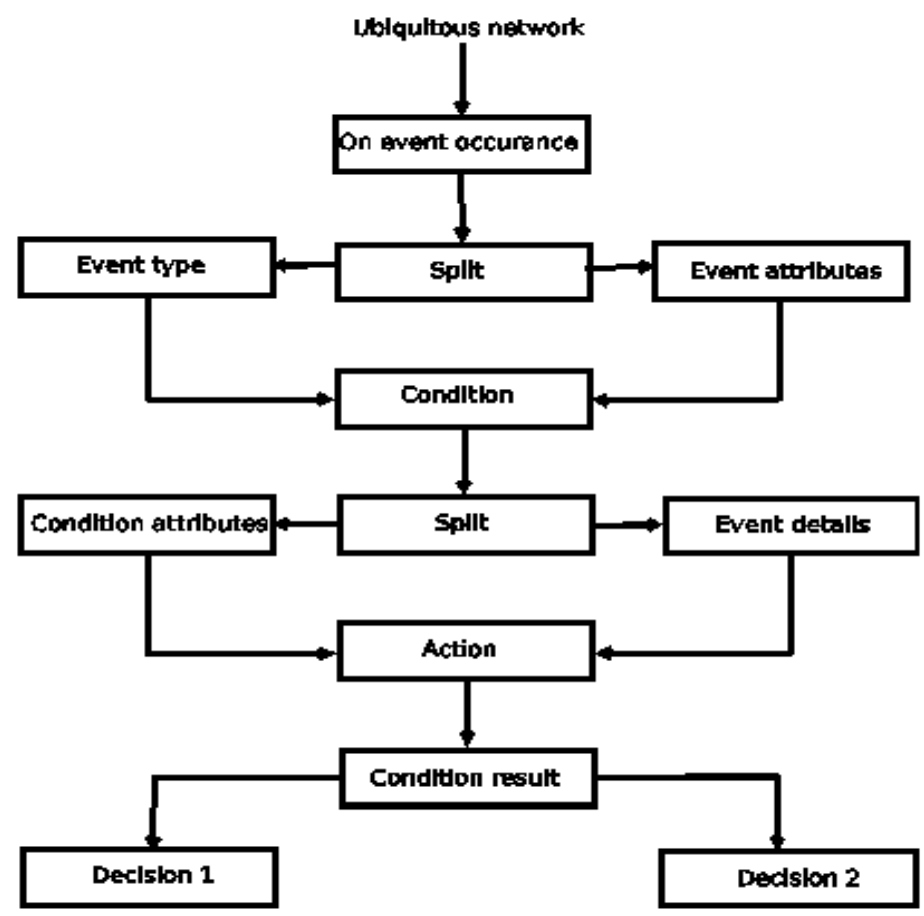

Figure 6. Event processing in ubiquitous network

\subsection{ECA scheme based Routing in Ubiquitous Network}

Table 6. Event(E1): Prepare route request and Event type(t1): Request

\begin{tabular}{|l|l|l|}
\hline \multicolumn{2}{|c|}{ Event attributes $\left(\mathrm{a}_{\mathrm{k}}\right)$} \\
\hline GUID & \multicolumn{1}{c|}{ Parameters } & \multicolumn{1}{c|}{ Values } \\
\hline datetime & Event unique ID & 9 \\
\hline int & Date\&time of event occurred & $10-03-2015$ at 1:00pm \\
\hline bool & Packet type & RREQ=1 \\
\hline bool & Join flag & Set J=1 or 0 \\
\hline bool & Repair flag & Set $\mathrm{R}=1$ or 0 \\
\hline bool & Gratuitous flag & Set $\mathrm{G}=1$ or 0 \\
\hline int & Destination only flag & Set $\mathrm{D}=1$ or 0 \\
\hline int & Hop count & Initial value=0 \\
\hline string*ip_address & Unique RREQ ID & 4 \\
\hline int & Destination IP address & 10.32 .21 .1 \\
\hline string*ip_address & Destination sequence \# & 13 \\
\hline int & Source IP address & 10.32 .21 .83 \\
\hline
\end{tabular}


Table 7. Condition $(\mathrm{C} 1)$ : Event attributes $\left(\mathrm{d}_{\mathrm{x}}\right)$ are obtained from event module

\begin{tabular}{|c|l|l|l|}
\hline \multicolumn{5}{|c|}{ Condition attributes $\left(\mathrm{c}_{\mathrm{m}}\right)$} \\
\hline \multicolumn{1}{|c|}{ Condition types } & \multicolumn{1}{|c|}{ Arguments } & \multicolumn{1}{c|}{ Operators } & \multicolumn{1}{c|}{ Results } \\
\hline $\begin{array}{l}\text { Condition1: } \\
\text { Checking valid path }\end{array}$ & Status & $?:$ & $\begin{array}{l}\text { No valid path exist toward } \\
\text { destination node }\end{array}$ \\
\hline $\begin{array}{l}\text { Condition2: Prepare } \\
\text { RREQ message and } \\
\text { setting sequence\# }\end{array}$ & Set & $+=$ & $\begin{array}{l}\text { Prepare RREQ message \& set } \\
\text { sequence number }+=1\end{array}$ \\
\hline
\end{tabular}

Table 8. Action(A1): Event and condition details are obtained

\begin{tabular}{|c|l|l|}
\hline \multicolumn{3}{|c|}{ Action attributes(at $\mathrm{p}_{\mathrm{p}}$} \\
\hline \multicolumn{1}{|c|}{ Event } & \multicolumn{1}{|c|}{ Condition result } & \multicolumn{1}{c|}{ Decision making } \\
\hline Prepare route request & $\begin{array}{l}\text { Node does not have route to } \\
\text { destination \&\&Prepare RREQ } \\
\text { and set seq } \#+=1\end{array}$ & $\begin{array}{l}\text { RRE message is prepared and } \\
\text { ready to broadcast }\end{array}$ \\
\hline
\end{tabular}

ECA Rule for Event(E1) : When\{Prepare route request event occurs\} If \{(Node does not have valid route) \&\& (Sequence number+=1)\} Then RREQ message is prepared and ready to broadcast

Table 9. Event(E2):Generate route reply and Event type(t2): Request

\begin{tabular}{|l|l|l|}
\hline \multicolumn{2}{|c|}{ Data types } & \multicolumn{1}{c|}{ Parameters } \\
\hline GUID & Event unique ID & \multicolumn{1}{c|}{ Values } \\
\hline datetime & Date\&time of event occurred & $11-04-2015$ at 2:00pm \\
\hline int & Packet type & RREP=2 \\
\hline bool & Repair flag & Set R=1 or 0 \\
\hline bool & Acknowledgement flag & Set A=1 or 0 \\
\hline bool & Prefix size & PS=00000 \\
\hline int & Hop count & Initial value=0 \\
\hline string*ip_address & Destination IP address & 10.32 .21 .83 \\
\hline int & Destination sequence \# & 2 \\
\hline string*ip_address & Source IP address & 10.32 .21 .1 \\
\hline time_t & Lifetime & $25 \mathrm{msec}$ \\
\hline
\end{tabular}


Table 10. Condition $(\mathrm{C} 2)$ : Event attributes $\left(\mathrm{d}_{\mathrm{x}}\right)$ are obtained from event module

\begin{tabular}{|l|l|l|l|}
\hline \multicolumn{1}{|c|}{ Condition attributes $\left(\mathrm{c}_{\mathrm{m}}\right)$} \\
\hline \multicolumn{1}{|c|}{ Condition types } & \multicolumn{1}{|c|}{ Orguments } & \multicolumn{1}{c|}{ Operators } & \multicolumn{1}{c|}{ Results } \\
\hline $\begin{array}{l}\text { Condition1:Destination } \\
\text { node }\end{array}$ & Status & $\begin{array}{l}\text { Destination node that has } \\
\text { active route }\end{array}$ \\
\hline $\begin{array}{l}\text { Condition2:Sequence } \\
\text { number }\end{array}$ & Verify & $>$ & $\begin{array}{l}\text { Sequence number must be } \\
\text { greater than that contained in } \\
\text { RREQ message }\end{array}$ \\
\hline
\end{tabular}

Table 11. Action(A2): Event and condition details are obtained

\begin{tabular}{|l|l|l|}
\hline \multicolumn{3}{|c|}{ Action attributes(at $\left.t_{\mathrm{p}}\right)$} \\
\hline Event & Condition result & Decision making \\
\hline Generate route reply & $\begin{array}{l}\text { Destination node that has active } \\
\text { route \&\& sequence \# is greater } \\
\text { than RREQ }\end{array}$ & $\begin{array}{l}\text { Reverse route reply to source } \\
\text { node }\end{array}$ \\
\hline
\end{tabular}

ECA Rule for Event(E2): When \{Generate route reply event occurs\} If (Destination node that has active route) \&\& (Sequence\# $>$ RREQ message) $\}$ Then $\{$ Reverse route reply to source node

Table 12. Event(E3):Route link has been broken and Event type(t3): Notification

\begin{tabular}{|l|l|l|}
\hline \multicolumn{3}{|c|}{ Event attributes $\left(\mathrm{a}_{\mathrm{k}}\right)$} \\
\hline \multicolumn{1}{|c|}{ Data types } & \multicolumn{1}{c|}{ Parameters } & \multicolumn{1}{c|}{ Values } \\
\hline GUID & Event unique ID & 25 \\
\hline datetime & Date\&time of event occurred & $14-04-2015$ at 5:00pm \\
\hline int & Packet type & RERR=3 \\
\hline bool & No delete flag & Set N=1 or 0 \\
\hline int & Destination count & Initial value=0 \\
\hline string*ip_address & Destination IP address & 10.32 .21 .51 \\
\hline int & Destination sequence \# & 6 \\
\hline
\end{tabular}

Table 13. Condition(C3): Event attributes $\left(\mathrm{d}_{\mathrm{x}}\right)$ are obtained from event module

\begin{tabular}{|c|l|l|l|}
\hline \multicolumn{4}{|c|}{ Condition attributes $\left(\mathrm{c}_{\mathrm{m}}\right)$} \\
\hline Condition type & \multicolumn{1}{|c|}{ Argument } & \multicolumn{1}{c|}{ Operator } & Result \\
\hline Condition1:Link fail & Check & $?:$ & Invalidate the route \\
\hline
\end{tabular}


Table 14. Action(A3): Event and condition details are obtained

\begin{tabular}{|l|l|l|}
\hline \multicolumn{3}{|c|}{ Action attributes $\left(\mathrm{at}_{\mathrm{p}}\right)$} \\
\hline Event & Condition result & Decision making \\
\hline Route link has been broken & Invalidate the route & List the affected nodes \\
\hline
\end{tabular}

ECA Rule for Event(E3): When \{Route link has been broken event occurs $\}$ If $\{$ Invalidate the route Then $\{$ List the affected nodes $\}$

Table 15. Event(E4):Generating route reply-ack and Event type(t4): Request

\begin{tabular}{|l|l|l|}
\hline \multicolumn{2}{|c|}{ Event attributes $\left(\mathrm{a}_{\mathrm{k}}\right)$} & \multicolumn{1}{c|}{ Parameters } \\
\hline GUID & \multicolumn{1}{|c|}{ Values } \\
\hline datetime & Event unique ID & 14 \\
\hline int & Date\&time of event occurred & $21-04-2015$ at 2:00pm \\
\hline bool & Packet type & RREP-ACK=4 \\
\hline bool & Repair flag & Set R=1 or 0 \\
\hline bool & Acknowledgement flag & Set A=1 or 0 \\
\hline int & Prefix size & PS=00000 \\
\hline string*ip_address & Hop count & Initial value=0 \\
\hline int & Destination IP address & 10.32 .21 .88 \\
\hline string*ip_address & Destination sequence \# & 7 \\
\hline time_t & Source IP address & 10.32 .21 .13 \\
\hline
\end{tabular}

Table 16. Condition(C4): Event attributes $\left(\mathrm{d}_{\mathrm{x}}\right)$ are obtained from event module

\begin{tabular}{|l|l|l|l|}
\hline \multicolumn{3}{|c|}{ Condition attributes $\left(\mathrm{c}_{\mathrm{m}}\right)$} \\
\hline \multicolumn{1}{|c|}{ Condition type } & \multicolumn{1}{|c|}{ Argument } & \multicolumn{1}{c|}{ Operator } & \multicolumn{1}{c|}{ Result } \\
\hline $\begin{array}{l}\text { Condition1:Acknowledgement } \\
\text { flag }\end{array}$ & Set & $=$ & $\begin{array}{l}\text { Use bidirectional link if } \\
\mathrm{A}=1,\end{array}$ \\
\hline
\end{tabular}

Table 17. Action(A4): Event and condition details are obtained

\begin{tabular}{|l|l|l|}
\hline \multicolumn{3}{|c|}{ Action attributes( $\left.\mathrm{at}_{\mathrm{p}}\right)$} \\
\hline Event & Condition result & Decision making \\
\hline Generating route reply-ack & Use bidirectional link if $\mathrm{A}=1$, & Send route reply-ack \\
\hline
\end{tabular}

ECA Rule for Event(E4): When $\{$ Generating route reply-ack\} If $\{$ Bidirectional link flag $A=1\}$ Then $\{$ List the affected nodes $\}$ 


\subsection{ECA state transition diagram}

- Event transition changes from one state to another state when initiated by a triggering event or condition.

- $\quad$ State machine is given by pentuple i.e. $\mathrm{SM}=\left(\sum, \mathrm{S}, \mathrm{s}_{0}, \delta, \mathrm{F}\right)$.

- $\sum$ is the input which is considered as set of events $\sum=\left\{E_{i}\right\}$.

- $\mathrm{S}$ is the \#of states i.e. state of a node $\mathrm{S}=\{1,2, \ldots, \mathrm{n}\}$.

- $\mathrm{s}_{0}$ is the initial state, where an event has occurred.

- $\delta$ be the transition which is considered as condition.

- $\delta=\left\{\right.$ State $_{\text {(old) }} \rightarrow$ State $_{(\text {new) }}$, Input $_{\text {(condition) }} \rightarrow$ Output $\left._{\text {(condition) }}\right\}$.

- $\mathrm{F}$ is the final state, where an action will be taken.

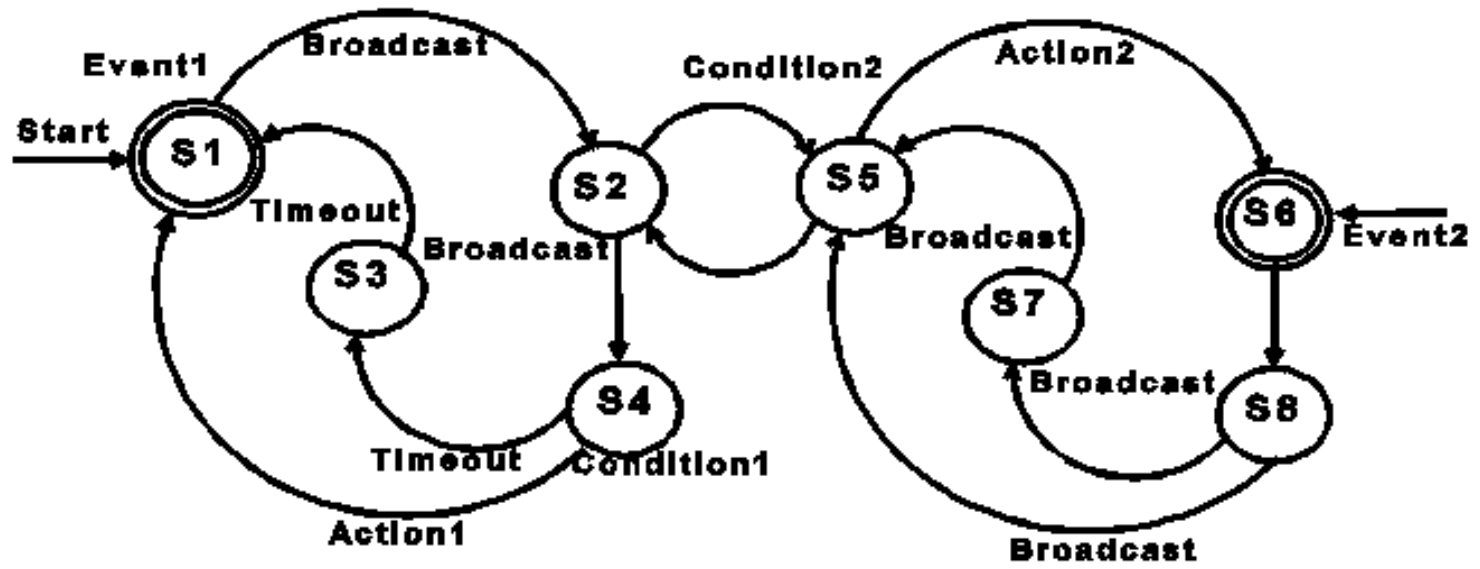

Figure 7. State transition diagram of an ECA Scheme

\section{CASE STUDY : ECA SCHEME IN UBIQUITOUS MUSEUM ENVIRONMENT}

Table 18. ECA scheme in ubiquitous museum environment

\begin{tabular}{|l|l|l|}
\hline \multicolumn{2}{|c|}{ Event } & \multicolumn{1}{|c|}{ Condition } \\
\hline $\begin{array}{l}\text { User is looking for a route to } \\
\text { visit exhibit }\end{array}$ & $\begin{array}{l}\text { (Interest=Science) } \\
\& \&(\text { Preference }=\text { Biology) }\end{array}$ & $\begin{array}{l}\text { Provide route information about } \\
\text { biology exhibit }\end{array}$ \\
\hline Lunch time & $\begin{array}{l}\text { (Preference=North-Indian) \&\& } \\
\text { (Time }>1 \text { PM })\end{array}$ & Route to restaurant \\
\hline High temperature in museum & Temperature $\geq 30^{0} \mathrm{C}$ & Switch on AC \\
\hline User blood pressure is low & BP <90/60 & Provide shortest route to hospital \\
\hline $\begin{array}{l}\text { User is spending more time in } \\
\text { front of exhibit }\end{array}$ & $\begin{array}{l}\text { User's history says user is new } \\
\text { to museum }\end{array}$ & $\begin{array}{l}\text { Provide details information about } \\
\text { exhibit }\end{array}$ \\
\hline
\end{tabular}




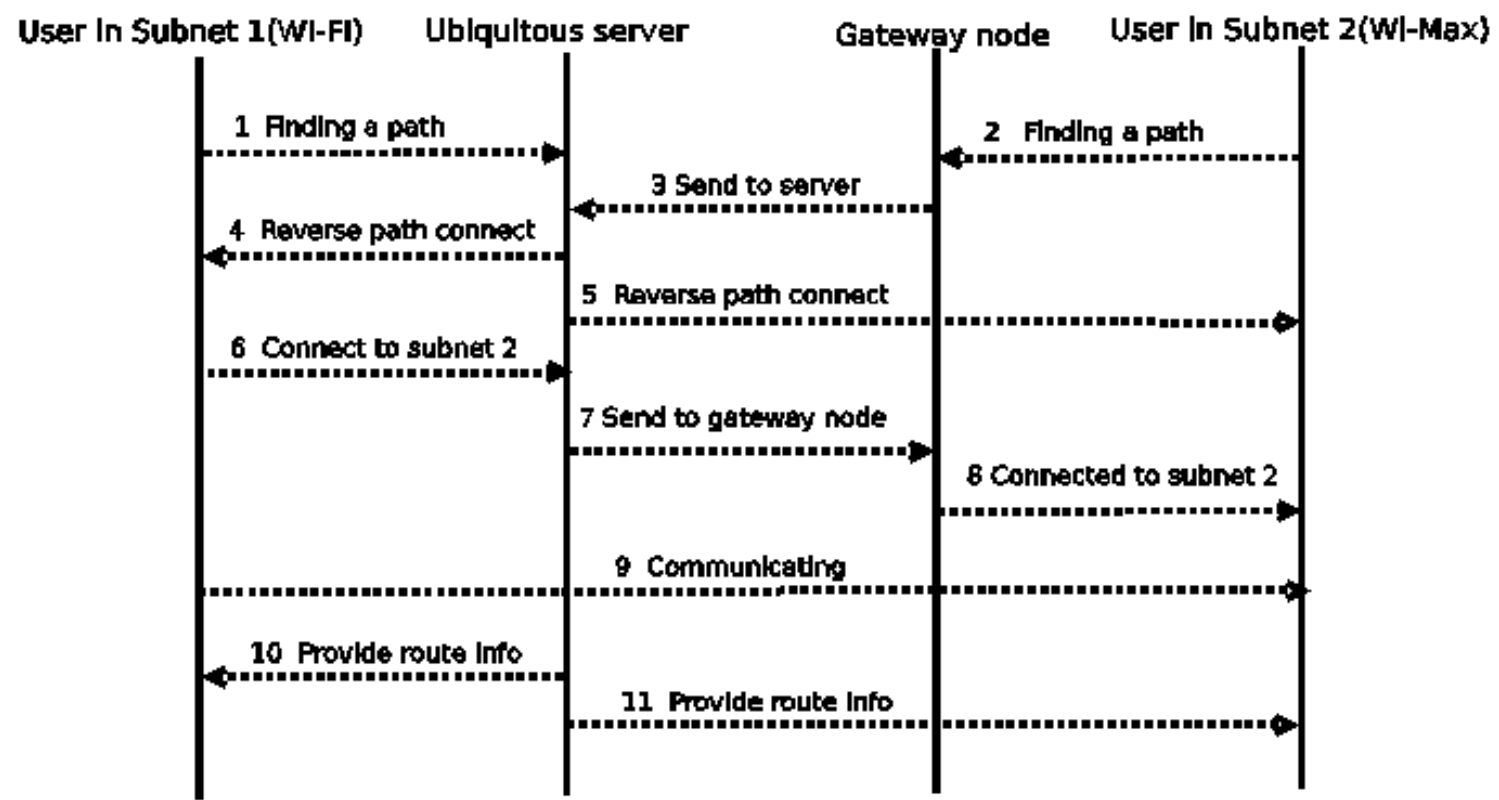

Figure 8. Sequence diagram to establish path between user and ubiquitous server

\section{SIMULATION ENVIRONMENT}

Proposed ECA scheme is implemented using C programming language and simulated in NS 2.34 simulator, by considering simulation parameters as depicted in table below. We have consider two different subnets such as Wi-Fi, MANETs in which random nodes are created and reverse path is established from ubiquitous server to the ubiquitous user, routing information is provided to the individual user based on interest, preferences.

Table 19. Simulation parameters

\begin{tabular}{|l|l|}
\hline \multicolumn{2}{|c|}{ Simulation parameters } \\
\hline Parameters & Values \\
\hline Nodes & 100 \\
\hline Routing protocol & ECA-AODV \\
\hline Transmission range & $30 \mathrm{mts}$ \\
\hline Simulation time & $500 \mathrm{~s}$ \\
\hline Topology size & $25 \mathrm{mX} 25 \mathrm{~m}$ \\
\hline Packet size & 512 Bytes \\
\hline Mobility & Random \\
\hline
\end{tabular}




\section{Simulation RESUltS}

ECA scheme is simulated using NS 2.34 simulator and set of results are obtained as shown in the following figures. In fig \#9, we have created 50 random nodes and simulated for $500 \mathrm{~ms}$ for AODV and ECA-AODV protocol, ECA-AODV protocol achieve lower packet delivery latency than normal AODV protocol. Fig \#10 in which 100 random nodes are created, different events are consider as an input for simulation by using 2 different routing protocols such as AODV and ECA-AODV respectively, after result execution, we conclude that data packet sending ratio of ECA-AODV is good in comparison with conventional AODV as and when event occur in the UbiNet.

In fig \#11, we have shown mobility speed versus control byte transferred over data byte delivered with normal routing and ECA based routing. Fig \#12 describes ECA scheme based RREQ and RREP message speed is high i.e. quicker response as and when event occur than the normal route request, route reply message and finally, fig \#13 explain number of event processed per second by the node in ECA-AODV is better as and when number of node increases compared to the conventional AODV routing protocol.

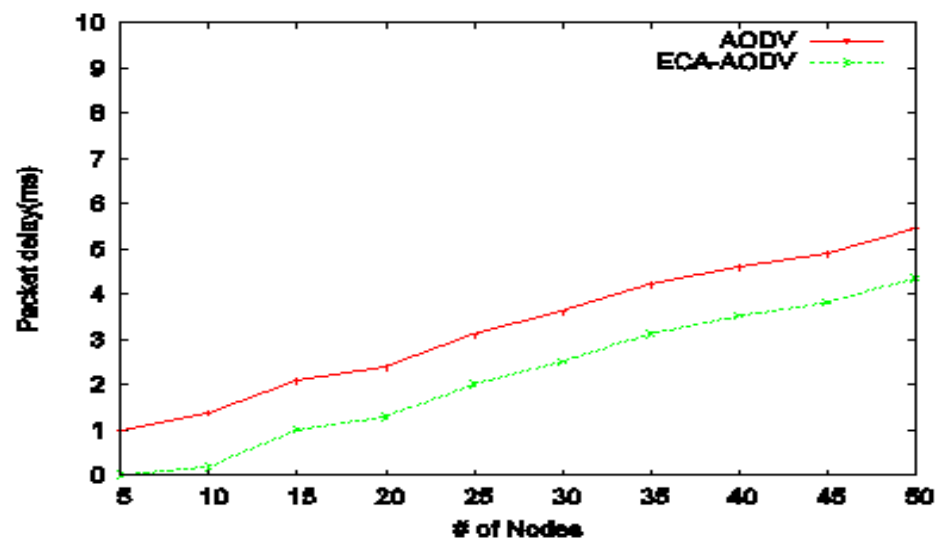

Figure 9. \#of nodes in network Vs packet delivery

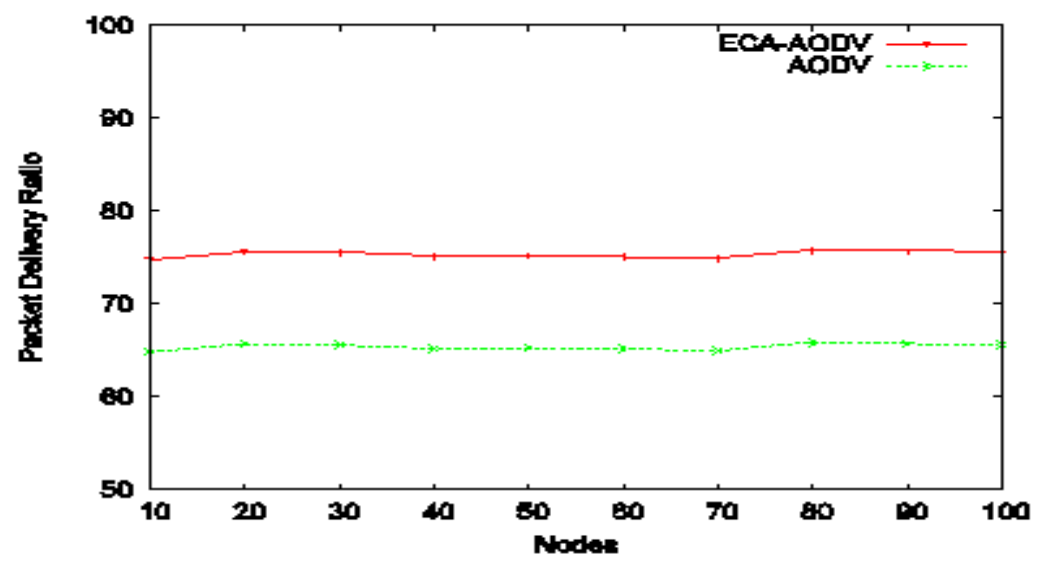

Figure 10. \#of nodes Vs data packet delivery ratio 


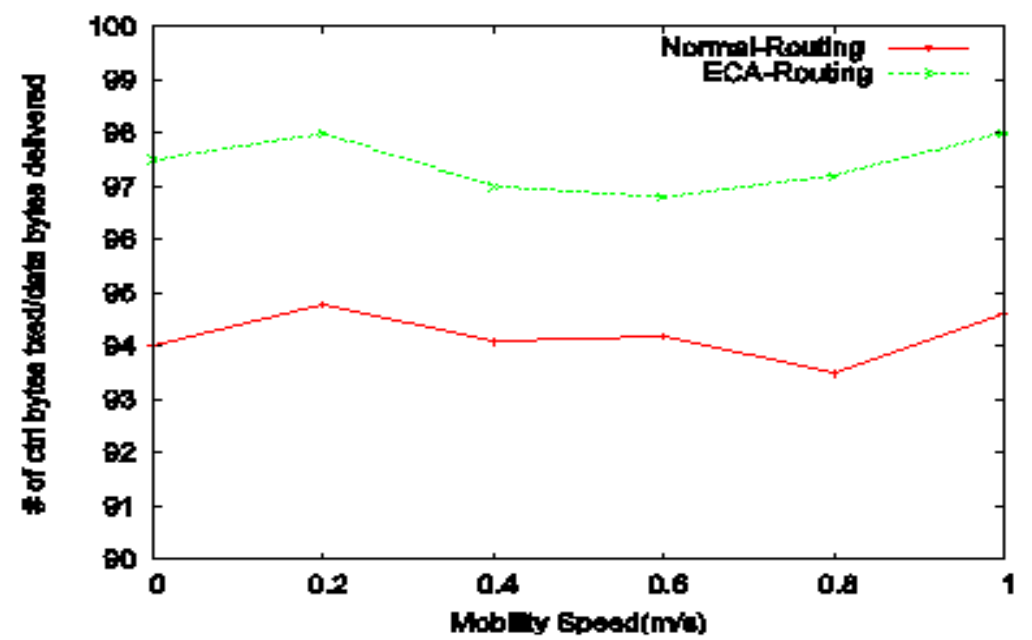

Figure 11. Mobility speed Vs \#of ctrl bytes transferred/data bytes delivered

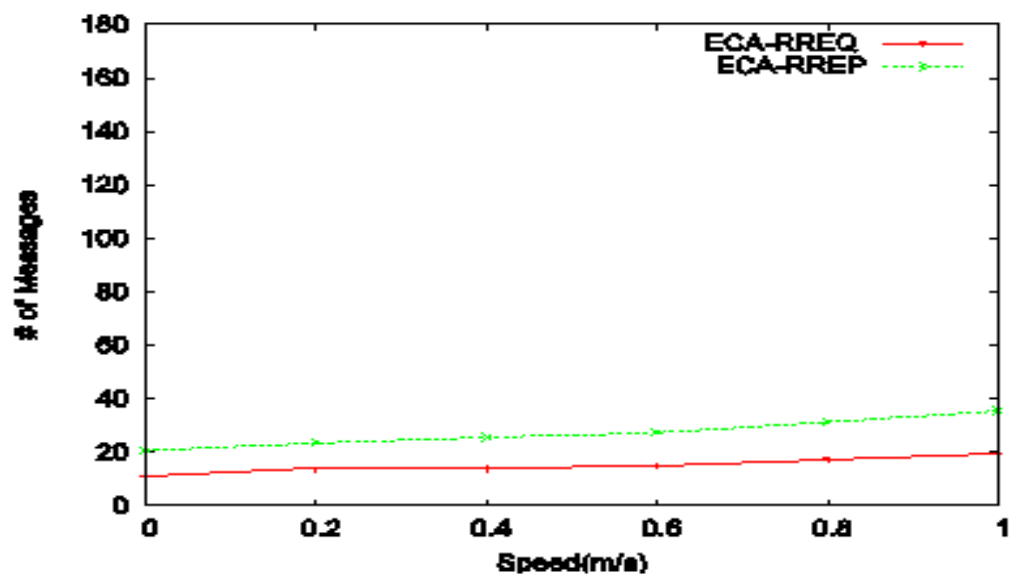

Figure 12. Speed Vs \#of messages

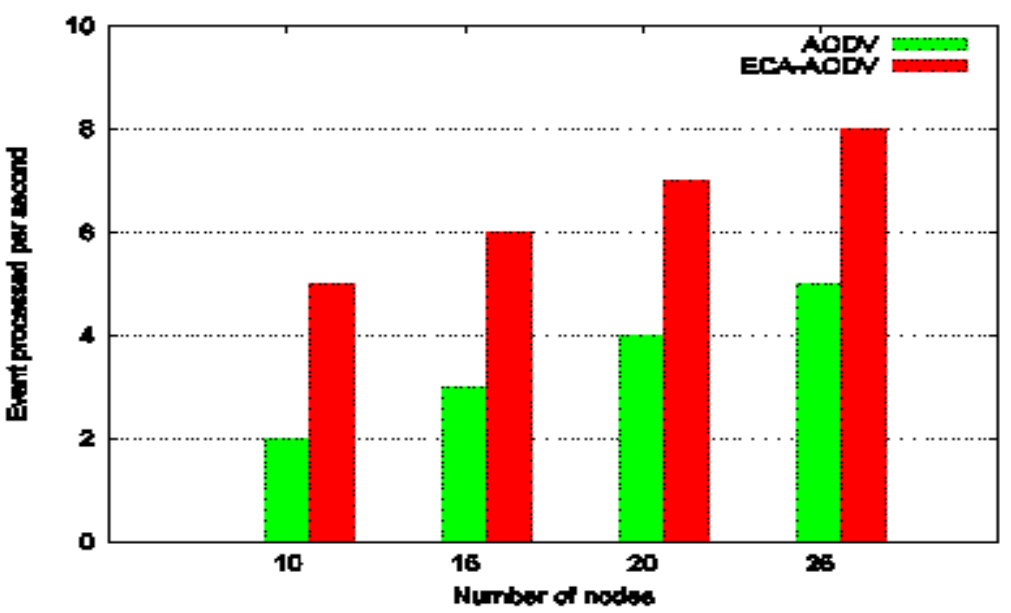

Figure 13. \#of nodes Vs event processed per second 


\section{CONCLUSIONS}

We have designed and simulated routing protocol using a novel ECA scheme in a UbiNet. Event module is designed using 2-tuples consists of event types and event attributes, condition module is designed using 2-tuples consists of event details and condition attributes and finally, an action module is designed using 1-tuple. We have considered ubiquitous museum environment as a case study to show the simulation of our proposed scheme, in which ubiquitous server provide uninterrupted connectivity and routing related information to the user as and when user move from one subnet to another subnet. However the proposed ECA scheme is flexible to supports dynamic network conditions in heterogeneous subnet and hence scheme is more effective as well as efficient as compared to non-ECA scheme in terms of parameters such as flexibility during runtime, easily adapt to the network dynamicity, quicker response as soon as event occur and easily adaptable to the types of network access technology used.

\section{ACKNOWLEDGMENTS}

We would like to thank our colleagues at Protocol Engineering and Technology unit, Department of ECE, Indian Institute of Science, Bangalore, India for their help and anonymous reviewers for their constructive and most valuable suggestions on improving the quality of the paper.

\section{REFERENCES}

[1] Chawathe, Y.; McCanne, S.; Brewer, E.A., "RMX: reliable multicast for heterogeneous networks," INFOCOM 2000. Nineteenth Annual Joint Conference of the IEEE Computer and Communications Societies. Proceedings. IEEE, vol.2, no., pp.795, 804 vol.2, 2000.

[2] T. Murakami and A. Fujinuma. Ubiquitous networking towards a new paradigm, Nomura research institute. April 2000.

3] Wang, H., Zhang, Y., \& Cao, J. (2006, May). Ubiquitous computing environments and its usage access control. In Proceedings of the 1st international conference on Scalable information systems (p. 6). ACM.

[4] Ge, M., Krishnamurthy, S. V., and Faloutsos, M. (2006). Application versus network layer multicasting in ad hoc networks: the ALMA routing protocol. Ad Hoc Networks, 4(2), 283-300.

[5] Young - Guk Ha; Joo - Chan Sohn; Young - Jo Cho, "ubiHome: An Infrastructure for Ubiquitous Home Network Services," Consumer Electronics, 2007. ISCE 2007. IEEE International Symposium on, vol., no., pp.1,6, 20-23 June 2007.

[6] Lee, Sung-Ju, William Su, and Mario Gerla. "On-demand multicast routing protocol in multi-hop wireless mobile networks." Mobile Networks and Applications 7.6 (2002): 441- 453.

[7] Orriëns, Bart, Jian Yang, and Mike P. Papazoglou. "Model driven service composition." ServiceOriented Computing -ICSOC 2003. Springer, Berlin Heidelberg, 2003. 75- 90.

[8] Yin, Liuguo, Changmian Wang, and Geir E. Øien. "An energy - efficient routing protocol for event driven dense wireless sensor networks." International Journal of Wireless Information Networks 16.3 (2009): 154-164 
[9] M Weiser. The computer of the 21st century. In ACM SIGMOBILE. Mobile computing and Communications. Pages 3-11, July 1999

[10] Levina, Olga, and Vladimir Stantchev. "A Model and an Implementation Approach for Event-Driven Service Orientation." International Journal on Advances in Software 2.2 and 3 (2009): 288-299

[11] Bhandari, Shiddartha Raj, and Neil W. Bergmann. "An internet-of-things system architecture based on services and events." Proceedings of the 2013 IEEE 8th International Conference on Intelligent Sensors, Sensor Networks and Information Processing: Sensing the Future, ISSNIP 2013. Vol. 1. IEEE, 2013

[12] Ye, Yan, Zhibin Jiang, Xiaodi Diao, and Gang Du. "Extended event-condition -action rules and fuzzy Petri nets based exception handling for workflow management." Expert Systems with Applications 38, no. 9 (2011): 10847-10861.

[13] Obweger, Hannes, Josef Schiefer, Martin Suntinger, and Peter Kepplinger. "Model-driven rule composition for event-based systems." International Journal of Business Process Integration and Management 5, no. 4 (2011): 344-357.

[14] Alferes, José Júlio, Federico Banti, and Antonio Brogi. "An event-condition-action logic programming language." Logics in Artificial Intelligence. Springer Berlin, Heidelberg, 2006. 29- 42.

[15] Bur, K.; Ersoy, C., "Quality-of-service-aware multicast routing in heterogeneous networks with Ad hoc extensions," Computer and Information Sciences, 2008. ISCIS '08. 23rd International Symposium on , vol., no., pp.1,6, 27-29 Oct. 2008

[16] Heo, Jungil, and Wooshik Kim. "Ad-hoc routing Protocol-based Ubiquitous Network System In the Hospital Environment." In Antennas, Propagation \& EM Theory, 2006. ISAPE'06. 7th International Symposium on, pp. 1-4. IEEE, 2006.

[17] C. E.Perkins and E. M.Royer. The ad hoc on-demand distance vector routing protocol. In Charles E.Perkins editor, Ad hoc networking Addison-Wesley, 2000

[18] C. Perkins, E. Belding-Royer, and S. Das. Ad hoc on-demand distance vector routing protocol in www.ietf.org/rfc/rfc3561, July 2003

[19] Zarei, M., Faez, K., \& Nya, J. M. (2008, December). Modified Reverse AODV routing algorithm using route stability in mobile ad hoc networks. In Multitopic Conference,2008. INMIC 2008. IEEE International (pp. 255-259).

[20] P. Venkataram and M. Bharath. Context based service discovery for ubiquitous applications. Information Networking(ICOIN), 2011 International conference on , pages 311-316, Jan 2011 


\section{AUTHORS}

Chandrashekhar Pomu Chavan received his BE in Computer Science and Engineering from Guru Nanak Dev Engineering College, Bidar, Karnataka, India, and M.Tech Degree in Network and Internet Engineering from Sri Jayachamarajendra College of Engineering, Mysore, Karnataka, India, in 2005 and 2008 respectively. Currently he is pursuing his Ph.D degree on Ubiquitous Network under the guidance of Prof. Pallapa Venkataram in the Department of Electrical Communication Engineering at Indian Institute of Science, Bangalore, India. His research interests are in the areas of Ubiquitous Computing, Pervasive Computing, Mobile Ad hoc Network, Context Aware System, and Routing Protocols.

Prof. PallapaVenkataram received his Ph.D. Degree in Information Sciences from the University of Sheffield, England, in 1986. He is currently the chairman for center for continuing education, and also a Professor in the Department of Electrical Communication Engineering, Indian Institute of Science, Bangalore, India. Dr. Pallapa's research interests are in the areas of Wireless Ubiquitous Networks, Social Networks, Communication Protocols, Computation Intelligence applications in Communication Networks and Multimedia Systems. He is the holder of a Distinguished Visitor Diploma from the Orrego University, Trujillo, PERU. He has published over 200 papers in International/national Journals/conferences. Written

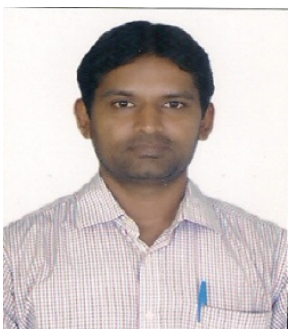
three books: Mobile and wireless application security, Tata McGraw-Hill, Communication Protocol Engineering, Prentice-Hall of India (PHI), New Delhi, 2004 (Co-author: Sunil Manvi), and Multimedia: Concepts \& Communication, Darling Kinderley(India) Pvt. Ltd., licensees of Pearson Education in South Asia, 2006. Edited two books: Wireless Communications for Next Millennium, McGraw-Hill, 1998, and Mobile Wireless Networks \& Integrated Services, John Wiley \& Sons(Asia) Pvt. Ltd., 2006(Co-editors: L.M.Patnaik \& Sajal K. Das). Written chapters for two different books, and a guest editor to the IISc Journal for a special issue on Multimedia Wireless Networks. He has received best paper awards at GLOBECOM'93 and INM'95 and also CDIL (Communication Devices India Ltd) for a paper published in IETE Journal. He is a Fellow of IEE (England), Fellow of IETE (India) and a senior member of IEEE Computer Society. 